\% Science and Engineering Research Council

Ti Rutherford Appleton Laboratory

$\underset{\pi}{\frac{1}{\varkappa}}$ Chilton DIDCOT Oxon OX11 0QX RAL-91-046

\title{
Relativistic Langmuir Waves \\ Generated by Ultra-Short Pulse Lasers
}

R Bingham, U de Angelis, M R Amin, R A Cairns and B McNamara 
Science and Engineering Research Council

"The Science and Engineering Research Council does not accept any responsibility for loss or damage arising from the use of information contained in any of its reports or in any communication about its tests or investigations" 


\title{
Relativistic Langmuir Waves Generated by Ultra-Short Pulse Lasers
}

\author{
R Bingham, ${ }^{*} \quad$ U de Angelis, ${ }^{*} \quad$ M R Amin,${ }^{\dagger} \mathrm{R}$ A Cairns, ${ }^{\dagger}$ \\ and B McNamara ${ }^{\ddagger}$
}

\begin{abstract}
The nonlinear interaction of ultra-short pulse high brightness lasers with a uniform plasma is studied numerically in 1-D. Apart from the usual nonlinear steepening and periodic lengthening of the plasma wave modification of the laser pulse also occurs if the pulse has a relatively long trailing edge compared to the leading edge. This induced modulation results in the formation of spikes on the laser pulse which are coincident with the Langmuir wave density maximum.
\end{abstract}

\section{Introduction}

Recently attention has focused on charged particle acceleration in a plasma by a fast, large amplitude, longitudinal electron plasma wave. The plasma beat wave and plasma wakefield accelerators are two efficient ways of producing ultra-high accelerating gradients. Starting with the plasma beat wave accelerator (PBWA) and laser wakefield accelerator (LWFA) schemes proposed by Tajima and Dawson ${ }^{[1]}$ in 1979 and the plasma wakefield accelerator (PWFA) also proposed by Dawson ${ }^{[2]}$ and co-workers in 1985, steady progress has been made in theory, simulations and experiments.

In the beat wave accelerator, two laser beams of nearly equal frequencies resonantly beat in a plasma in such a way that their frequency and wavenumber differences correspond to the plasma wave frequency and wavenumber. For co-propagating laser beams

"Rutherford Appleton Laboratory, Chilton, Didcot, Oxon, OX11 0QX, U.K.

tUniversity of St. Andrew, Fife, KY16 9SS, U.K.

'Leabrooke Computing, Oxford, U.K. 
the plasma wave phase velocity $v_{p h}=\left(\omega_{1}-\omega_{2}\right) /\left(k_{1}-k_{2}\right) \simeq \omega_{p e} / k_{p}$ is equal to the group velocity of the laser beams which is slightly less than $c$, the velocity of light, if the laser frequencies are much greater than the plasma frequency. A very similar mechanism is involved in the stimulated Raman scattering process. The group velocity of the plasma wave is essentially zero for a cold plasma, so that the excited plasma wave is left behind as the laser pulse propagates through the plasma. The laser pulse therefore always propagates into regions of undisturbed plasma leaving a large amplitude plasma wave behind which will eventually evolve into plasma turbulence.

Electrons which are injected with velocities close to the phase velocity of the plasma wave can be trapped and accelerated to higher energies. A significant experiment on the plasma beat wave accelerator was carried out by Joshi's group at UCLA ${ }^{[3]}$ where an accelerating field of $1 \mathrm{GeV} / \mathrm{m}$ was demonstrated, similar fields have since been reported by the Imperial College and RAL group ${ }^{[4]}$, using a neodimium glass laser and similar plasma density's to that used by the UCLA group.

In the LWFA, a short pulse of laser light, whose frequency is much greater than the plasma frequency, excites a wake of plasma oscillations due to the ponderomotive force. Since the plasma wave is not resonantly driven as in the beat wave accelerator the plasma density does not have to be of a high uniformity to achieve large amplitude waves. With the rapid development of high brightness lasers such as the $10 \mathrm{~J}, 1$ ps (Tabletop Ten Terawatt) system developed by the LLNL group ${ }^{[5]}$, which could eventually be the front end of $1 \mathrm{~kJ}, 1 \mathrm{ps}$ (Petawatt) system, the LWFA is a promising source of energetic particles of the order of $10-100 \mathrm{GeV}$ in distances of the order of a meter. The focal intensities of such lasers will be $\geq 10^{19} \mathrm{~W} / \mathrm{cm}^{2}$ with $v_{\text {ose }} / c \geq 1$, where $v_{\text {ose }}$ is the electron quiver velocity in the laser field, which is the strong nonlinear relativistic regime of the LWFA, any analysis must therefore be in the strong relativistic regime.

The PWFA on the other hand does not require the use of lasers only a high current but low energy relativistic electron beam, which is injected into a cold plasma. As in the two-stream instability, the streaming electrons lose energy to the background plasma by exciting a plasma wave. The phase velocity of the plasma wave is tied to the velocity of the injected electrons, which is close to $c$. The concept of the PWFA has been known since the late 50's when theory and experiments were carried out by the Soviets ${ }^{[6,7]}$. Because of the need for ultra-relativistic beams no detailed studies, however, were carried out until recently by Dawson ${ }^{[2]}$ and his group at UCLA, with experiments being carried out at Argonne National Laboratory [8,9]. The results of these experiments have directly verified the existence of electron acceleration in plasma wakefields by the injection of a test bunch of electrons into a beam-driven plasma wave. The experiments validated relevant predictions of linear wavefield theory such as the 
excitation and structure in both the longitudinal and transverse directions of the excited plasma wave. A number of drawbacks associated with the plasma beatwave scheme have to be overcome (a) the necessity for very uniform plasma (b) plasma wave saturation due to relativistic frequency detuning (c) the resonance condition requires fine laser tuning. Plasma waves excited by either short pulse lasers (PLWA) or by charged particle beams (PWFA) appear to be easier and more efficient than the beat wave excitation. In the wakefield scheme since the excitation is due to a single pump all the problems raised by the resonance condition are absent and the plasma, wave can grow to larger amplitudes.

Numerical solution of model equations describing laser wakefield excitation.

Using a model based on the one fluid, cold relativistic hydrodynamics and Maxwell equations together with a "quasi-static" approximation a set of two coupled nonlinear equations describing the self-consistent evolution in 1-D of the laser pulse vector potential envelope and the scalar potential of the excited wake-field are derived. Starting from the equation for electron momentum

$$
\frac{\partial \mathbf{p}}{\partial t}+v_{z} \frac{\partial \mathbf{p}}{\partial z}=-\left(e \mathbf{E}+\frac{1}{c} \mathbf{v} \times \mathbf{B}\right)
$$

where

$$
\mathbf{p}=m_{0} \gamma \mathbf{v}, \gamma=\left(1+p^{2} / m_{0}^{2} c^{2}\right)^{1 / 2}
$$

$m_{0}$ and $\mathbf{v}$ being the electron rest mass and velocity.

In eq. (1) we have assumed that all quantities only depend on $z$ and $t, z$ being the direction of propagation of the (external) pump and

$$
\mathbf{E}=-\frac{1}{c} \frac{\partial \mathbf{A}_{\perp}}{\partial z}-\hat{z} \frac{\partial \phi}{\partial z} ; \mathbf{B}=\nabla \times \mathbf{A}_{\perp} ; \quad \mathbf{A}_{\perp}=\hat{x} A_{x}+\hat{y} A_{y},
$$

Where $\mathbf{A}_{\perp}$ is the vector potential of the electromagnetic pulse and $\phi$ the ambipolar potential due to charge separation in the plasma.

Using eqs. (1) and (2) the perpendicular component of the electron momentum is found to be

$$
\frac{p_{\perp}}{m_{0} c}=\frac{e}{m_{0} c^{2}} \mathbf{A}_{\perp} \equiv \mathrm{a}(z, t)
$$

and we can write 


$$
\gamma=\left[1+\left(\frac{p_{\perp}}{m_{0} c}\right)^{2}+\left(\frac{p_{z}}{m_{0} c}\right)^{2}\right]^{1 / 2} \equiv \gamma_{a} \gamma_{\|}
$$

where

$$
\gamma_{a}=\left(1+\mathbf{a}^{2}\right)^{1 / 2} ; \gamma_{\|}=\left(1-\beta^{2}\right)^{-1 / 2}
$$

and $\beta=v_{z} / c$

The equations derived from this model are now the longitudinal component of eq. (1), the equation of continuity, Poisson's equation and the wave equation for $a(z, t)$, which are given by

$$
\begin{gathered}
\frac{1}{c} \frac{\partial}{\partial t}\left(\gamma_{a} \sqrt{\gamma_{\|}^{2}-1}\right)+\frac{\partial}{\partial z}\left(\gamma_{a} \gamma_{\|}\right)=\frac{\partial \phi}{\partial z} ; \varphi \equiv \frac{e \phi}{m_{0} c^{2}} \\
\frac{1}{c} \frac{\partial n}{\partial t}+\frac{\partial}{\partial z}\left(n \frac{\sqrt{\gamma_{\|}^{2}-1}}{\gamma_{\|}}\right)=0 \\
\frac{\partial^{2} \varphi}{\partial z^{2}}=\frac{\omega_{p 0}^{2}}{c^{2}}\left(\frac{n}{n_{0}}-1\right) \\
c^{2} \frac{\partial^{2} \mathbf{a}}{\partial z^{2}}-\frac{\partial^{2} \mathbf{a}}{\partial t^{2}}=\omega_{p 0}^{2} \frac{n}{n_{0}} \frac{\mathbf{a}}{\gamma_{a} \gamma_{\|}} .
\end{gathered}
$$

Assuming a driving pulse of the form

$$
\mathbf{a}(z, t)=\frac{1}{2} \mathbf{a}_{0}(\xi, \tau) e^{-i \theta}+c . c .
$$

where $\theta=\omega_{0} t-k_{0} z, \omega_{0}$ and $k_{0}$ being the central frequency and wave-number, $\xi=$ $z-v_{g} t$ and $v_{g}=\partial \omega_{0} / \partial k_{0}$ is the group velocity and $\tau$ is a slow time-scale such that

$$
a_{0}^{-1} \frac{\partial^{2} a_{0}}{\partial \tau^{2}} \ll \omega_{0}^{2}
$$

accounting for changes in the pump due to the plasma reaction, the wave equation becomes 


$$
\begin{gathered}
{\left[2 \frac{\partial}{\partial \tau}\left(i \omega_{0} a_{0}+v_{g} \frac{\partial a_{0}}{\partial \xi}\right)+c^{2}\left(1-v_{g}^{2} / c^{2}\right) \frac{\partial^{2} a_{0}}{\partial \xi^{2}}+2 i \omega_{0}\left(\frac{c^{2} k_{0}}{\omega_{0}}-v_{g}\right) \frac{\partial a_{0}}{\partial \xi}\right] e^{-i \theta}+c . c .} \\
=\left[c^{2} k_{0}^{2}-\omega_{0}^{2}+\frac{n}{n_{0}} \frac{\omega_{p 0}^{2}}{\gamma_{a} \gamma_{\|}}\right] a_{0} e^{-i \theta}+c . c .
\end{gathered}
$$

where $\omega_{p 0}$ is the plasma frequency of the unperturbed plasma. Equations (6), (7), (8) and (11) form the basic set for this problem in the "envelope approximation".

A simplified set of equations for the weak pump, weakly-relativistic regime ie. $\left|a_{0}\right|^{2}<$ $1, \beta^{2} \ll 1$ was derived and solved by Gorbunov et al ${ }^{[10]}$ and Sprangle et al. ${ }^{[1]}$ The solution has the structure of a wake-field growing inside the e.m. pulse and oscillating behind the pulse with the maximum amplitude being reached inside the pulse. Using the quasi-static approximation the time derivative can be neglected in the electron fluid equations (6) and (7) yielding the following constants

$$
\begin{aligned}
& \gamma_{a}\left(\gamma_{\|}-\beta_{0} \sqrt{\gamma_{\|}^{2}-1}\right)-\varphi=1 \\
& n\left(\beta_{0} \gamma_{\|}-\sqrt{\gamma_{\|}^{2}-1}\right)=n_{0} \beta_{0} \gamma_{\|}
\end{aligned}
$$

where $\beta_{0}=v_{g} / c$. The constants of integration have been chosen in such a way that

$$
n=n_{0}, \gamma_{\|}=1, \varphi=0
$$

for

$$
\gamma_{a}=1\left(\left|a_{0}\right|^{2}=0\right)
$$

Using equation (12) and (13) general system (6)-(9) can be written as two coupled equations describing the evolution of the laser pulse envelope $a_{0}$ and the scalar potential $\varphi$

$$
\begin{gathered}
\frac{\partial^{2} \varphi}{\partial \xi^{2}}=\frac{\omega_{p 0}^{2}}{c^{2}} G \\
2 i \omega_{0} \frac{\partial a_{0}}{\partial \tau}+2 c \beta_{0} \frac{\partial^{2} a_{0}}{\partial \tau \partial \xi}+\frac{c^{2} \omega_{p 0}^{2}}{\omega_{0}^{2}} \frac{\partial^{2} a_{0}}{\partial \xi^{2}}=-\omega_{p 0}^{2} H a_{0}
\end{gathered}
$$


where

$$
G=\frac{\sqrt{\gamma_{\|}^{2}-1}}{\beta_{0} \gamma_{\|}-\sqrt{\gamma_{\|}^{2}-1}}, H=1-\frac{\beta_{0}}{\gamma_{a}\left(\beta_{0} \gamma_{\|}-\sqrt{\gamma_{\|}^{2}-1}\right)}
$$

The present set of nonlinear equations (15) and (16) are obtained using a quasistatic approximation, which yields two integrals of the motion given by equations (12) and (13). The model is valid for electromagnetic pulses of arbitrary polarization and intensities $\left|a_{0}\right|^{2} \geq 1$.

A simplified version of equation (15) for $\beta_{0}=1$ and $a_{0}=$ constant has been solved by Tsintsadze ${ }^{[12]}$ while the coupled system for $\beta_{0}=1$ has been solved by Bulanov et al ${ }^{[13]}$. Sprangle et al ${ }^{[14]}$ considered the solution of equation (15) together with an equation describing the full wave equation for a without the envelope approximation but still for the limiting case of $\beta_{0}=1$. A review of the different approaches and limitations can be found in de Angelis ${ }^{[15]}$ as well as a full derivation of the present model.

The set of coupled nonlinear equations (15) and (16) are solved numerically in the stationary frame of the pulse. Eq. (15), Poisson's equation for the wakefield, is solved with the initial conditions $\varphi=0, \frac{\partial \varphi}{\partial \xi}=0$ by a simple predictor-corrector method. The envelope equation (16) describing the evolution of the laser pulse is written as two coupled equations for the real and imaginary parts of $a_{0}$ and solved implicitly.

The present model based on a quasi-static approximation yields two integrals of motion equs. (12) and (13). The quasi-static approximation used states that ${ }^{[14]}$ if the laser pulse is sufficiently short, the fields $a_{o}$ and $\varphi$ are expected to evolve slowly during a transit time of the plasma throught the laser pulse. In this approximation the quantity $\gamma_{c}=\gamma_{a}\left(\gamma_{\|}^{2}-1\right)^{\frac{1}{2}}$ is a constant. The quantity $\gamma_{c}$ is checked continuously during the numerical calculation and it is found to change slowly with time, the greater $a_{o}$ the sooner $\gamma_{c}$ changes. Table 1 shows for different input parameters, the number of plasma periods after which $\gamma_{c}$ changes by $25 \%$. Clearly the quasi-static approximation is more valid for lower values of $a_{o}$ and $\omega_{\text {po }} / \omega_{o}$, this quantity is checked frequently during the calculation and is found to eventually change after a number of plasma periods, the greater $a_{0}$ the sooner $\gamma_{c}$ changes. Numerical solutions of equations 15 and 16 showing the evolution of the excited plasma wake-field potential $\varphi$ and electric field $E_{w}$ as well as the envelope of the laser pulse is shown in Figs 1 and 2 . In all the figures we have plotted the fields as normalized quantities $\varphi=e \phi / m_{0} c^{2}, E_{w}=e|E| / m_{0} c \omega_{p 0}$ and $\left|a_{0}\right|=\frac{e A_{0}}{m_{0} c^{2}}$, defined previously. The horizontal scale in all cases is the position $\xi=z-v_{g} t$ normalized by the plasma wavelength $\lambda_{p}$, with the pulse propagating from left to right, 
time is in terms of the plasma period, $T_{p}=2 \pi / \omega_{p 0},\left|a_{0}^{i n}\right|$ signifies the peak amplitude of the normalized initial vector potential of the lase pulse and finally $\sigma_{r}$ and $\sigma_{f}$ represent the Gaussian pulse rise an fall coefficients respectiveiy (for a symmetric pulse $\sigma_{\mathrm{r}}=\sigma_{f}$ ). The present study considers different pulse shapes. Figure 1 shows the evolution of $\left|a_{0}\right|, \varphi$ and the electric field $E_{w}$ at time $t=2 T_{p}$ for similar parameters considered by Tsintsadze ${ }^{[12]}$. It is obvious that the most efficient pulse shape for wavefield generation is the one with a steep leading edge and a long trailing edge. Figure 2 shows the evolution of $\left|a_{0}\right|, \varphi$ and $E_{w}$ at two different times, at late times there is significant distortion of the trailing edge of the laser pulse resulting in photon spikes. Figures 3 and 4 show the time development of the laser pulse vector potential $\left|a_{o}\right|$, both real and imaginary parts, the potential $\varphi$ and the normalized density perturbation $\delta n / n_{\circ}$ for a pulse with a sharp rise and a long trailing edge. The position of the amplitude spikes occurring on the laser pulse coincides with the maximum in $\delta n / n o$. This distortion occurs where the wake potential has a minimum and the density has a maximum. The spike arises as a result of the photons interacting with the plasma density inhomogeneity with some photons being accelerated (decelerated) as they propagate down (up) the density gradient this effect was predicted by Dawson is called the photon accelerator ${ }^{[16]}$. The distortion of the trailing edge increases with increasing $\omega_{p 0} / \omega_{0}$. For a square wave pulse, pulse distortion is found to occur at the leading edge, as shown by Bulanov et al $^{[13]}$ and also by our present simulations figure 5 . Finally we find that from figure 2 the longitudional potential $\frac{e \phi}{m c^{2}}>1$ or $\frac{e E_{x}}{m_{e} \omega_{p 0 c}}>1$, is significantly greater than fields abtained in the PBWA, which are limited by relativistic de-tuning, no such saturation exists in the LWFA, and the pulse distortion observed is equivalent to pulse compression in real space and therefore leads to frequency broadening.

\section{Conclusions}

An envelope equation describing in 1-D the evolution of an electromagnetic pulse together with the equation describing the wakefield potential solved in the stationary frame of the pulse without the restriction of $\beta_{o}=1$. The solutions show a strong distortion effect on the E.M. pulse, namely the generation of a short intensity spike which coincides with the maximum of the plasma density perturbation. This intensity spike will have the effect of broadening the frequency spectrum of the pulse. Various pulse shapes are solved for, with the most effective shape for wavefield generation being a sharp rise with a slowly falling tail.

\section{Acknowledgements}

This work was partly supported by an EC twinning grant between Rutherford Appleton Laboratory and the University of Naples. 


\section{REFERENCES}

1. T Tajima, J M Dawson, Phys. Rev. Lett., $\underline{43}, 267$ (1979)

2. P Chen, J M Dawson, R W Buff and T Katsouleas. Phys. Rev. Lett., $\underline{54}, 693$ (1985)

3. C E Clayton, C Joshi, C Darrow and D Umstadter. Phys. Rev. Lett., $\underline{54}, 2343$ (1985)

4. A E Dangor, A K L Dymoke-Bradshaw and A E Dyson. Physica Scripta, T30, $267(1990)$

5. C Darrow, M D Perry, F Patterson, E M Campbell, T Katsouleas and W B Mori. AIP Conf. Proc. 199 on Advanced Accelerator Concepts ed. C. Joshi.

6. Ya B Fainberg, Proceeding of CERN Symposium on High Energy Accelerators and Pion Physics, Geneva, (1956)

7. F Kharchenko, Ya B Fainberg, R M Nikoleav, E A Kornilov, E A Lutsenko and N S Pedenko.Sov. Phys. J.E.T.P., 11, 493 (1960)

8. J B Rosenzweig, D B Cline, B Cole, H Figueroa, W Gai, R Konecny, J Norem, P Schoessow and J Simpson. Phys. Rev. Lett., $\underline{61}, 98$ (1988)

9. J B Rosezweig, P Schoessow, B Cole, W Gai, R Konecny, J Norem and J Simpson. Phys. Rev. A., $\underline{39},(1989)$

10. L Gorbunov and V Kirsanov. Sov Phys JETP, $\underline{66}, 290,(1987)$

11. P Sprangle, E Esarey, E Ting and G Joyce. App. Phys. Lett., $\underline{53}, 2146,(1988)$

12. N L Tsintsadze, Physica Scripta, T30, 41, (1990)

13. S V Bulanov, V I Kirsanov and A S Sakharov, Physica Scripta, T30, 208 (1990)

14. P Sprangle, E Esarey and A Ting, Phys. Rev. Lett., 64, 2011, (1990).

15. U de Angelis, Physica Scripta, $\underline{\text { T30, }} 210$ (1990)

16. S C Wilks, J M Dawson, W B Mori, T Katsouleas and M E Jones. Phys. Rev. Lett. 62, 2600, (1989). 
Table 1

\begin{tabular}{|c|c|c|c|}
\hline & $\left|a_{0}^{i n}\right|$ & $\omega_{p o} / \omega_{o}$ & $\begin{array}{l}\text { Time } t \text { in terms of plasma } \\
\text { period } T_{p}=2 \pi / \omega_{p o}\end{array}$ \\
\hline \multirow{5}{*}{$\begin{array}{l}\sigma_{r}=0.25 \lambda_{p} \\
\sigma_{f}=0.25 \lambda_{p}\end{array}$} & 1 & 0.05 & 40 \\
\hline & & 0.01 & 250 \\
\hline & 2 & 0.05 & 30 \\
\hline & & 0.10 & 15 \\
\hline & 3 & 0.05 & 25 \\
\hline \multirow{5}{*}{$\begin{array}{l}\sigma_{\mathrm{r}}=0.25 \lambda_{p} \\
\sigma_{f}=1.50 \lambda_{p}\end{array}$} & 1 & 0.05 & 30 \\
\hline & & 0.01 & 200 \\
\hline & 2 & 0.05 & 25 \\
\hline & & 0.10 & 10 \\
\hline & 3 & 0.05 & 20 \\
\hline \multirow{5}{*}{$\begin{array}{l}\sigma_{r}=1.50 \lambda_{p} \\
\sigma_{f}=0.25 \lambda_{p}\end{array}$} & 1 & 0.05 & 50 \\
\hline & & 0.01 & 250 \\
\hline & 2 & 0.05 & 50 \\
\hline & & 0.10 & 20 \\
\hline & 3 & 0.05 & 50 \\
\hline Square & & 0.01 & 250 \\
\hline pulse width & 2 & 0.05 & 40 \\
\hline$\ell_{p}=1.0 \lambda_{p}$ & & 0.10 & 6 \\
\hline
\end{tabular}




\section{Figure Captions}

1. The values of the magnitude of the normalised vector potnetial $\left|a_{0}\right|$ (solid curves) and scalar potential $\varphi$ and/or wake-electric field $E_{\omega}$ (dashed curves) with position $\xi=z-v_{g} t,\left|a_{0}^{i n}\right|=\sqrt{ } 8, \omega_{p 0} / \omega_{0}=0.01, t=2 T_{p}$. (a) and (b) Gaussian rise and fall $\sigma_{r}=\sigma_{f}=5 c / \omega_{p 0}=0.795 \lambda_{p} ;(c)$ and (d) Gaussian rise $\sigma_{r}=c / \omega_{p 0}=0.159 \lambda_{p}$, Gaussian fall $\sigma_{f}=9 c / \omega_{p 0}=1.433 \lambda_{p} ;(\mathrm{e})$ and (f) Gaussian rise $\sigma_{r}=9 c / \omega_{p 0}=$ $1.433 \lambda_{p}$, Gaussian fall $\sigma_{f}=9 c / \omega_{p 0}=0.159 \lambda_{p}$. $T_{p}=2 \pi / \omega_{p o}$, is the plasma period and $\lambda_{p}=2 \pi c / \omega_{p o}$, is the plasma wavelength.

2. The values of the magnitude of the normalised vector potential $\left|a_{0}\right|$ (solid curves) and scalar potnetial $\Phi$ and/or wake-electric field $E_{w}$ (dashed curves) with position $\xi=z-v_{g} t,\left|a_{0}^{i n}\right|=2, \omega_{p 0} / \omega_{0}=0.1$. Gaussian rise $\sigma_{r}=0.25 \lambda_{p}$, Gaussian fall $\sigma_{f}=1.5 \lambda_{p}$, Curves (a) and (b) are at time $t=0.2 T_{p} ;(\mathrm{c})$ and (d) are at $t=10 T_{p}$.

3. The values of the magnitude of the normalized vector potentiasl $\left|a_{o}\right|$ (solid curve) (the real and imaginary parts) and the scalar potential $\varphi$ with position $\xi=z-v_{g} t$. For a Gaussian rise $\sigma_{r}=0.25 \lambda_{p}$, Gaussian fall $\sigma_{f}=1.5 \lambda_{p}, \omega_{p o} / \omega_{o}=0.05\left|a_{o}^{i n}\right|=2$. Curves (a) and (b) are for $t=0.4 T_{p}$, (c) and (d) are for $t=20 T_{p}$, (e) and (f) are for $t=40 T_{p}$.

4. Evolution of the narmalized density perturbation for the same conditions used in figure 3. Curves (a), (b) and (c) are for the same times also ie $t=0.4 T_{p}, 20 T_{p}$ and $40 T_{p}$ respectively.

5. The values of $\left|a_{o}\right|^{2} / 2$ (curve 1) and the potential $\varphi$ (curve 2) for square pulse-width $1_{p}=1.0 \lambda_{p}$ and $\left|a_{o}^{\text {in }}\right|=2$. (a) and (b) are for $\omega_{p o} / \omega_{o}=0.01,(\mathrm{c})$ and (d) are for $\omega_{p o} / \omega_{o}=0.05,(e)$ and (f) are for $\omega_{p o} / \omega_{o}=0.1$. Curve (a) is at time $t=2 T_{p}$, (b) is at $t=100 T_{p}$, (c) is at $t=0.4 T_{p}$, (d) is at $t=20 T_{p}$, (e) is at $t=0.2 T_{p}$ and (f) is at $t=10 T_{p}$. 
Fig1
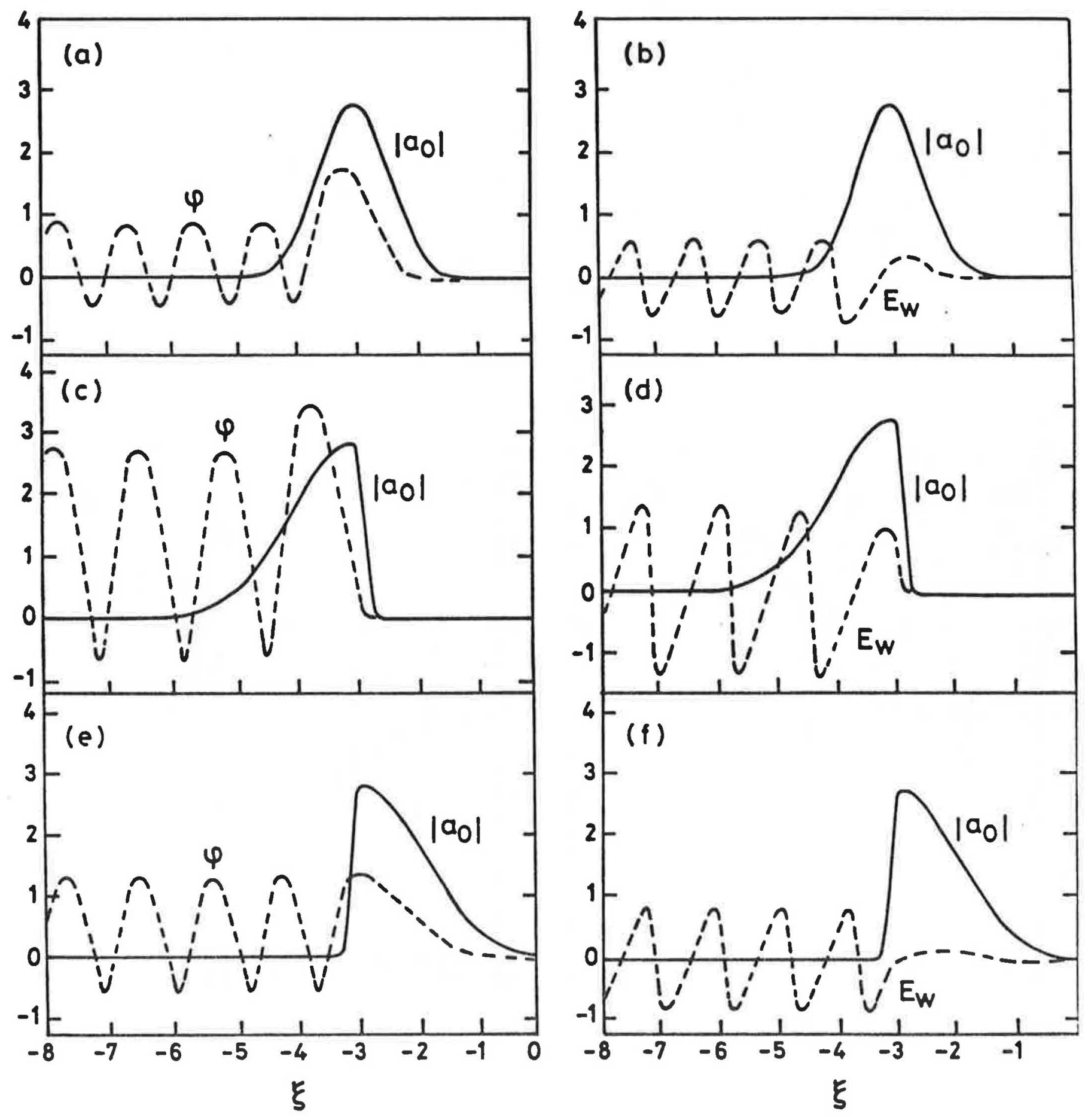
Fig 2
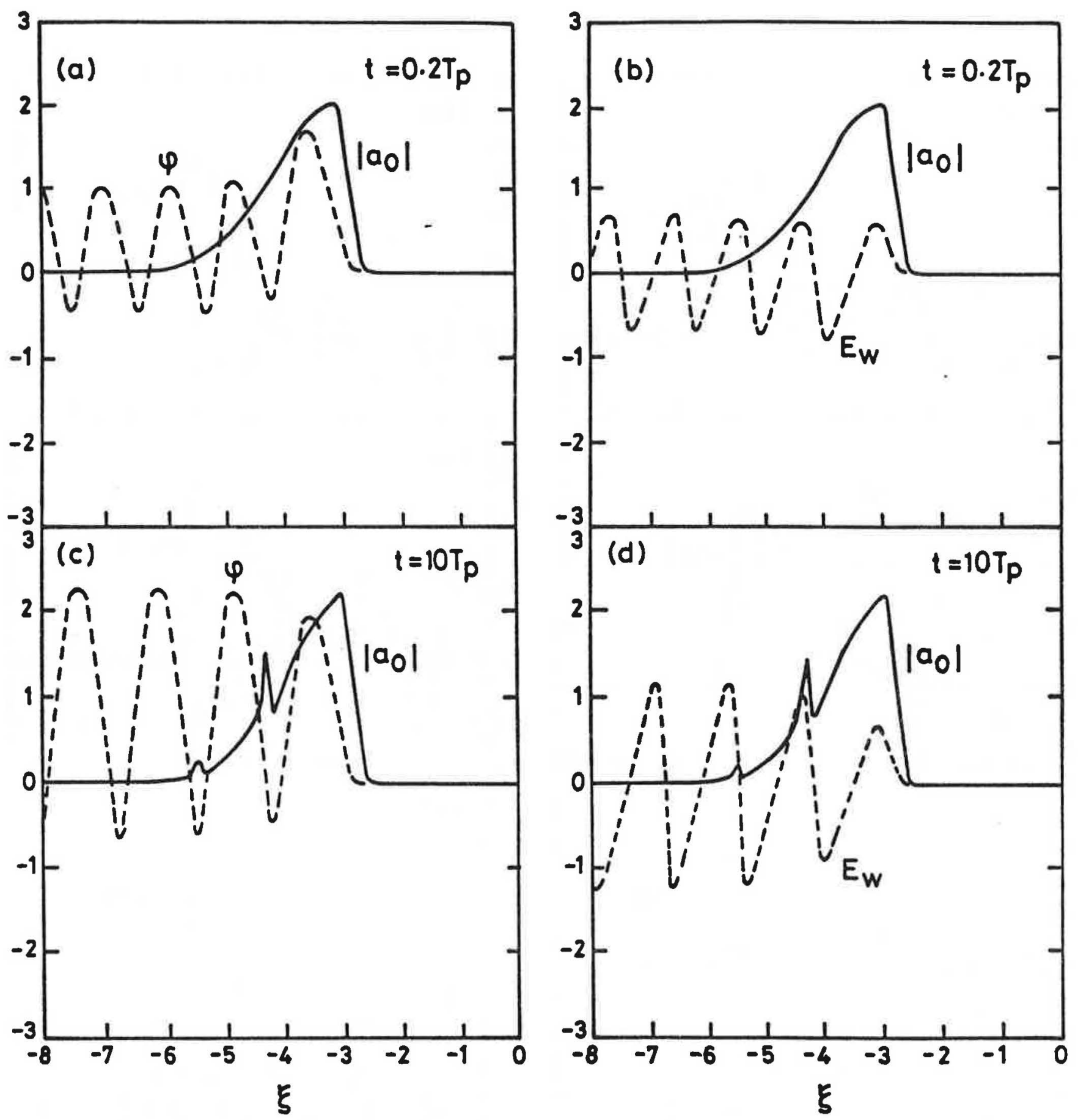
Figi
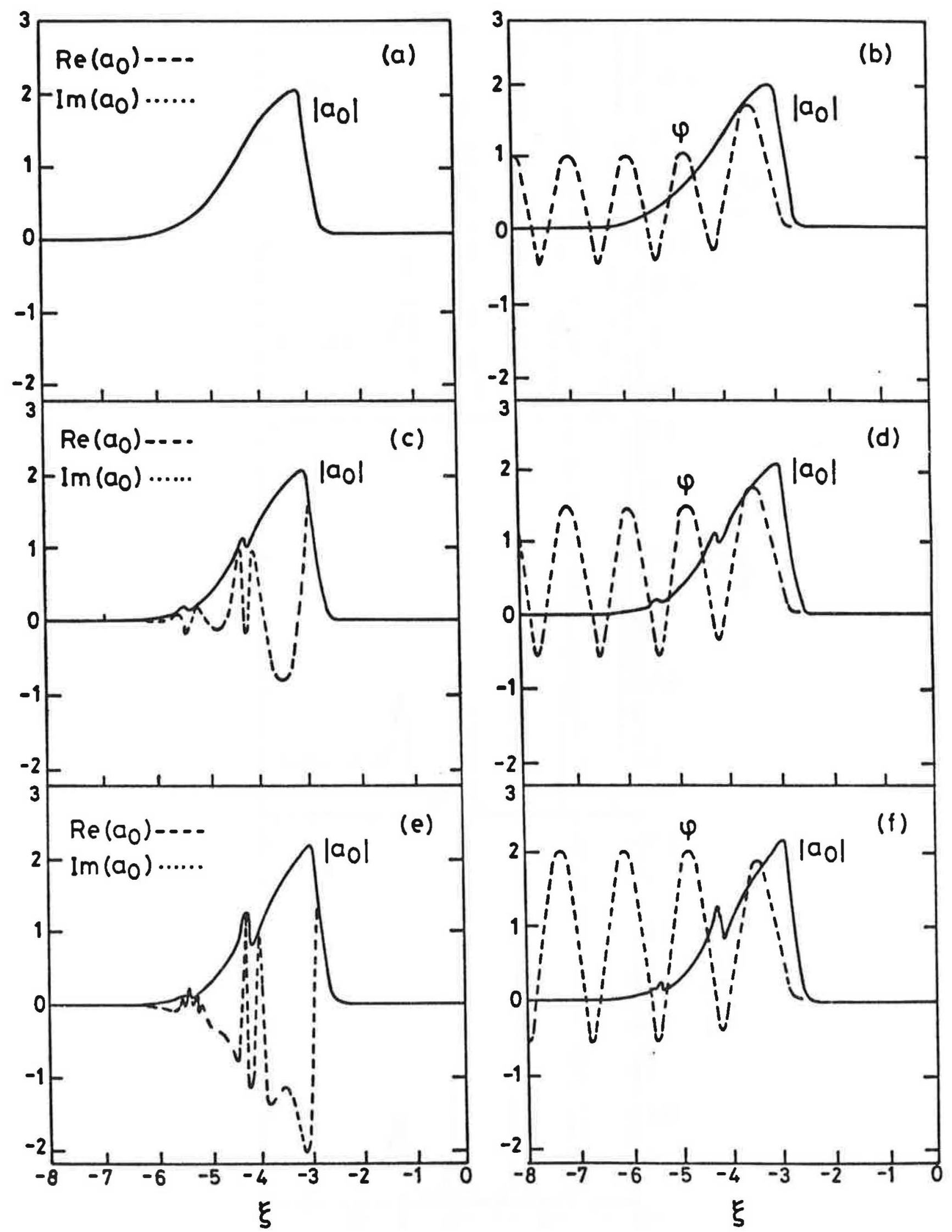


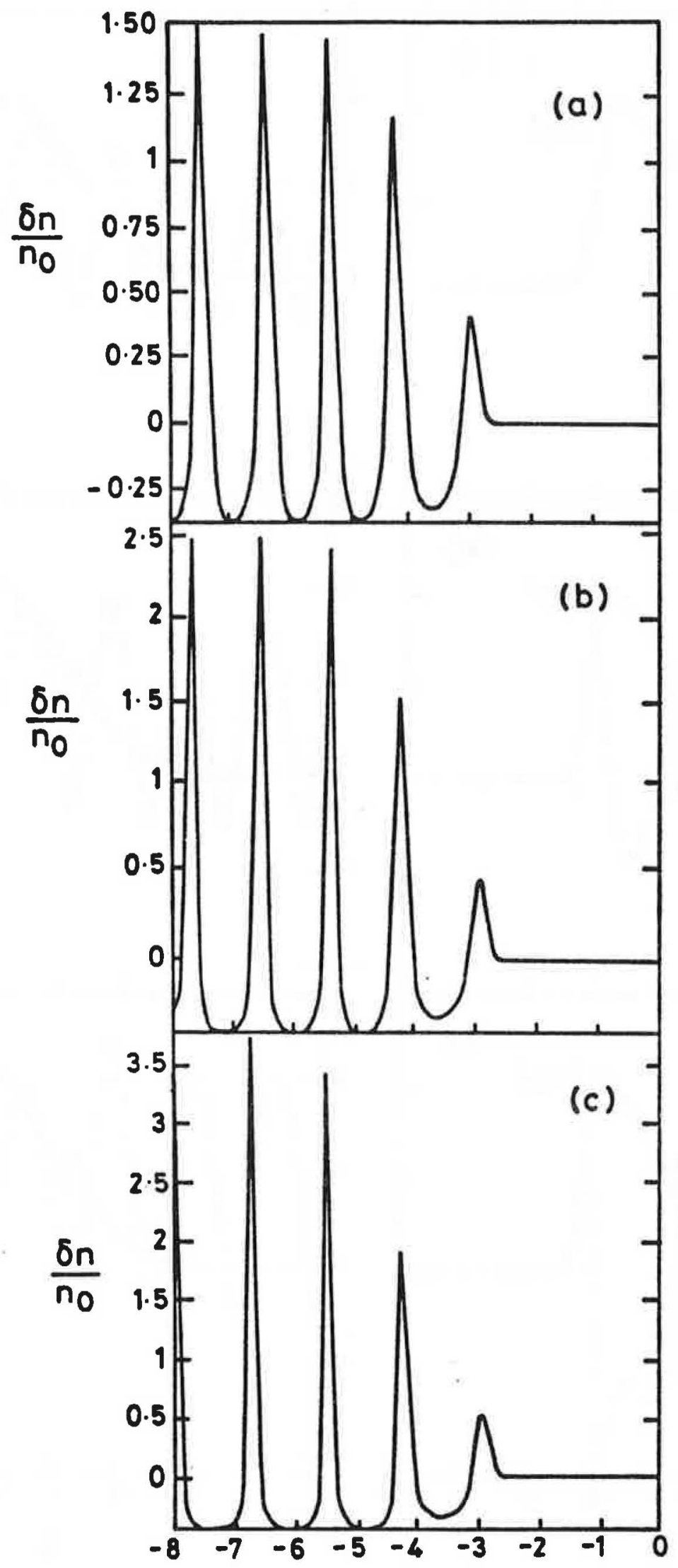


Fy
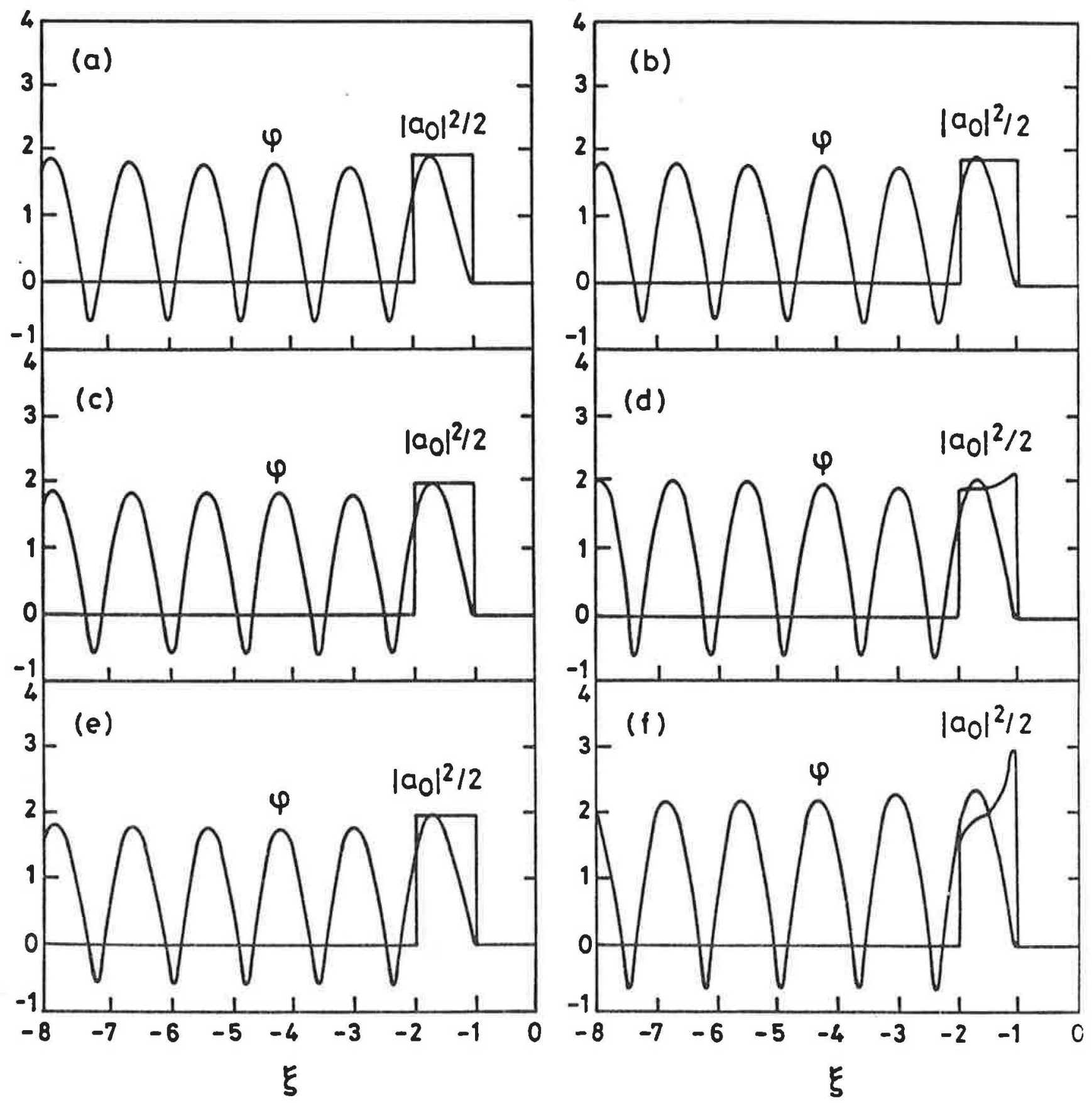

